\title{
Stability of linear systems with time-varying delays using Bessel-Legendre inequalities
}

\author{
Alexandre Seuret and Frédéric Gouaisbaut
}

\begin{abstract}
This paper addresses the stability problem of linear systems with a time-varying delay. Hierarchical stability conditions based on linear matrix inequalities are obtained from an extensive use of the Bessel inequality applied to Legendre polynomials of arbitrary orders. While this inequality has been only used for constant discrete and distributed delays, this paper generalizes the same methodology to time-varying delays. We take advantages of the dependence of the stability criteria on both the delay and its derivative to propose a new definition of allowable delay sets. It is shown that a light and smart modification in the definition of this set leads to relevant conclusions on the numerical results.

Keywords. Integral inequality, stability analysis, time-varying delay systems, allowable delay sets, linear matrix inequalities.
\end{abstract}

\section{INTRODUCTION}

This paper is devoted to the stability analysis of linear timedelay systems, which represent a wide class of systems arising in many applications such as in biology, in traffic control, in engineering, in cyber-physical systems or in networked control systems (see for instance the books and survey papers [2], [3], [7], [22]). Compared to the delay-free case, the difficulties when studying time-delay systems mainly come from the infinite dimensional nature of this class of systems, which require a dedicated analysis.

The aim of this paper is to provide stability conditions of time-delay systems where the delay is assumed to be a bounded continuous time-varying function with bounded derivatives. In the literature, there exist several major results on this topic, see for instance the Lyapunov-Krasovskii approach [24], [8], [15], [5], [23], [10], the "Input-to-State" or "Input -to-Output" approaches [6], which are related to the robust analysis [1], [11]. Focusing on the Lyapunov-Krasovskii approach, several techniques have been considered to reduce the conservatism of the stability conditions, often formulated in terms of Linear Matrix Inequalities (LMI). The conservatism of these LMIs, formulated in terms of delay upperbounds, generally comes from several manipulations allowing to express the infinite dimensional stability problem into a finite LMI test. A list of technical tools that have been considered in the literature to solve this problem includes matrix inequalities [13], the reciprocally convex combination lemmas [16], [27].

The objective of this paper is the design of generic stability conditions for linear systems subject to a continuous timevarying delay by using the Bessel-Legendre inequality. These conditions generalize the original works on Bessel-Legendre

*This work was supported by ANR project SCIDiS contract number 15CE23-0014.

A. Seuret and F. Gouaisbaut are with LAAS - CNRS, Université de Toulouse, CNRS, UPS, 7 av. du Colonel Roche, F-31400 Toulouse, France aseuret, fgouaiselaas.fr inequality for systems with constant (discrete or distributed) delay presented in [19], [21] to the case of time-varying delays. This work is also a generic extension of the stability conditions for time-varying delay systems of former papers which use the Wirtinger-based inequality [18], the auxiliarybased integral inequalities [17], or the free-weighting matrix integral inequality from [25]. We also stress that another contribution of this paper is to evaluate the impact of the allowable delay set characterizing the delay functions as suggested in [20]. Several numerical experiments show that the proposed Lyapunov-Krasovskii functional is very competitive if an adequate delay set is constructed. It suggests then that the conservatism induced by the method stems mainly from the robust analysis performed on the LMI.

Notations: Throughout the paper $\mathbb{R}^{n}$ denotes the $n$ dimensional Euclidean space with vector norm $|\cdot|, \mathbb{R}^{n \times m}$ is the set of all $n \times m$ real matrices. The notation $P \succ 0$, for $P \in \mathbb{R}^{n \times n}$, means that $P$ is symmetric and positive definite. The set $\mathbb{S}^{n}\left(\mathbb{S}_{+}^{n}\right)$ represents the set of symmetric (positive definite) matrices of $\mathbb{R}^{n \times n}$. The set of continuous functions from an interval $\mathcal{I} \subset \mathbb{R}$ to $\mathbb{R}^{n}$ which are square integrable is denoted as space $\mathcal{L}_{2}\left(\mathcal{I}, \rightarrow \mathbb{R}^{n}\right)$. The symmetric matrix $\left[\begin{array}{cc}A & B \\ * & C\end{array}\right]$ stands for $\left[\begin{array}{cc}A & B \\ B^{T} & C\end{array}\right]$. $\operatorname{diag}(A, B)$ stands for the diagonal matrix $\left[\begin{array}{ll}A & 0 \\ 0 & B\end{array}\right]$. Moreover, for any square matrix $A \in \mathbb{R}^{n \times n}$, we define $\operatorname{He}(A)=A+A^{T}$. For any function $x:[-h,+\infty) \rightarrow \mathbb{R}^{n}$, $x_{t}(\theta)$ stands for $x(t+\theta)$, for all $t \geq 0$ and all $\theta \in[-h, 0]$.

\section{PROBLEM FORMULATION}

\section{A. System data}

Consider a linear time-delay system of the form:

$$
\left\{\begin{array}{llrl}
\dot{x}(t) & =A x(t)+A_{d} x(t-h(t)), & & \forall t \geq 0, \\
x(t) & =\phi(t), & & \forall t \in\left[-h_{2}, 0\right],
\end{array}\right.
$$

where $x(t) \in \mathbb{R}^{n}$ is the state vector, $\phi$ is the initial condition function, $A$ and $A_{d}$ are constant matrices and $h_{2}$ is a given positive scalar. The delay function is assumed to be continuous and differentiable and satisfies the following constraint

$$
\forall t \geq 0, \quad(h(t), \dot{h}(t)) \in \mathcal{H} \subset \mathbb{R}^{+} \times \mathbb{R},
$$

where $\mathcal{H}$ is assumed to be a convex bounded subset of $\mathbb{R}^{+} \times$ $\mathbb{R}$, denoted as the allowable delay set for system (1). In the literature, the set $\mathcal{H}$ is often selected as a polytope $\left[h_{1}, h_{2}\right] \times$ $\left[d_{1}, d_{2}\right]$, for some appropriate values of $h_{1}, h_{2}, d_{1}, d_{2}$. In this paper we define alternative polyhedral allowable delay sets with the same number of vertices. We will show that other possibilities can be considered and lead to notable changes in the numerical experiments reported in Section VI. 


\section{B. Legendre polynomials and their properties}

Let us first recall the definition of the Legendre polynomials, used in the definition of the Lyapunov-Krasovskii functional employed thereafter.

Definition 1: The "shifted" Legendre polynomials are the sequence of polynomials defined over the interval $[0,1]$, which are given for all $i \in \mathbb{N}, L_{i}(u)=(-1)^{i} \sum_{j=0}^{i} p_{j}^{i} u^{j}$ where $p_{j}^{i}=$ $(-1)^{j}\left(\begin{array}{c}i \\ j\end{array}\right)\left(\begin{array}{c}i+j \\ j\end{array}\right)$, and $\left(\begin{array}{l}k \\ l\end{array}\right)$ refers to the binomial coefficients given by $\frac{k !}{(k-l) ! l !}$.

In the sequel, the notation $\mathbb{L}_{N}$ refers to a polynomial matrix of dimension $(N+1) n \times n$, where $n$ and $N$ are positive integers, and is given by

$$
\mathbb{L}_{N}(u):=\left[\begin{array}{llll}
L_{0}(u) I_{n} & L_{1}(u) I_{n} & \ldots & L_{N}(u) I_{n}
\end{array}\right]^{T} .
$$

The orthogonality property of the Legendre polynomials can be summarized by the following statement. For any matrix $R$ in $\mathbb{S}_{+}^{n}$, it holds

$$
\forall N \in \mathbb{N}, \quad \int_{0}^{1} \mathbb{L}_{N}(u) R^{-1} \mathbb{L}_{N}^{T}(u) \mathrm{d} u=\tilde{R}_{N}^{-1}
$$

where $\tilde{R}_{N}=\operatorname{diag}\{R, 3 R, \ldots,(2 N+1) R\}$. In addition, the evaluation of these polynomials at the boundaries of the interval $[0,1]$ are simply given, for any integer $N \geq 0$, by

$$
\mathbb{L}_{N}(1)=\left[\begin{array}{c}
I_{n} \\
I_{n} \\
\vdots \\
I_{n}
\end{array}\right]:=\mathbf{1}_{\mathbf{N}}, \quad \mathbb{L}_{N}(0)=\left[\begin{array}{c}
I_{n} \\
-I_{n} \\
\vdots \\
(-1)^{N} I_{n}
\end{array}\right]:=\overline{\mathbf{1}}_{\mathbf{N}} .
$$

Since orthogonal polynomials usually verify recursive properties, Legendre polynomials satisfy the two following differentiation rules, that will be the key technical tools of this paper

$$
\begin{array}{ll}
\frac{d}{d u} \mathbb{L}_{N}(u) & =\bar{\Gamma}_{N} \mathbb{L}_{N}(u)=\Gamma_{N} \mathbb{L}_{N-1}(u) \\
\frac{d}{d u}\left(u \mathbb{L}_{N}(u)\right) & =\mathbb{L}_{N}(u)+\Theta_{N} \mathbb{L}_{N}(u),
\end{array}
$$

where $\bar{\Gamma}_{N}=\left[\begin{array}{ll}\Gamma_{N} & 0_{n(N+1), n}\end{array}\right], \Gamma_{N}=\gamma_{N} \otimes I_{n}$ and $\Theta_{N}=$ $\theta_{N} \otimes I_{n}$, where $\otimes$ refers to the classical Kronecker product. Matrices $\gamma_{N} \in \mathbb{R}^{(N+1) \times N}$ and $\theta_{N} \in \mathbb{R}^{(N+1) \times(N+1)}$ are defined by

$$
\begin{aligned}
& \gamma_{N}(k, i)=\left\{\begin{array}{lll}
0 & \text { if } & k \geq i, \\
(2 k-1)\left(1-(-1)^{i+k}\right) & \text { if } & k<i .
\end{array}\right. \\
& \theta_{N}(k, i)=\left\{\begin{array}{lll}
0 & \text { if } & k>i, \\
k & \text { if } & k=i, \\
(2 k-1) & \text { if } & k<i .
\end{array}\right.
\end{aligned}
$$

These two derivation rules are derived from classical properties of the Legendre polynomials. Basically, it means that their derivative are expressed using Legendre polynomials of lower degree.

\section{From constant to time-varying delays}

In [19], a first stability analysis based on Legendre-based Lyapunov-Krasovskii functionals has been provided to cope with linear systems subject to constant time-delay. In this paper, the following functional has been employed

$$
\begin{aligned}
W_{N}\left(x_{t}, \dot{x}_{t}\right)= & \chi_{N}^{T}(t) P_{N} \chi_{N}(t)+\int_{t-h}^{t} x^{T}(s) S x(s) \mathrm{d} s \\
& +h \int_{t-h}^{t} \int_{\theta}^{t} \dot{x}^{T}(s) R \dot{x}(s) \mathrm{d} s \mathrm{~d} \theta,
\end{aligned}
$$

where $N \in \mathbb{N}, P_{N} \in \mathbb{S}^{(N+2) n}, S, R \in \mathbb{S}_{+}^{n}$ and the augmented vector $\chi_{N}^{T}(t)=\left[\begin{array}{ll}x_{t}^{T}(0) & h \phi_{N}^{T}(t)\end{array}\right]$ and

$$
\phi_{N}(t)=\frac{1}{h} \int_{-h}^{0} \mathbb{L}_{N}\left(\frac{s+h}{h}\right) x_{t}(s) \mathrm{d} s, \quad \forall N \geq 0 .
$$

Guided by this functional (7) dedicated to the analysis of systems with a constant delay, we propose to consider the following extension which aims at dealing with the case of time-varying delays. It consists in using the following Lyapunov-Krasovskii functional

$$
\begin{aligned}
& V_{N}\left(x_{t}, \dot{x}_{t}\right)=\tilde{x}_{N}^{T}(t) P_{N} \tilde{x}_{N}(t)+\int_{t-h}^{t} x^{T}(s) S x(s) \mathrm{d} s \\
& \quad+\int_{t-h_{2}}^{t-h} x^{T}(s) Q x(s) \mathrm{d} s+h_{2} \int_{t-h_{2}}^{t} \int_{\theta}^{t} \dot{x}^{T}(s) R \dot{x}(s) \mathrm{d} s \mathrm{~d} \theta,
\end{aligned}
$$

where, in this case, the matrix $P_{N}$ is now in $\mathbb{S}^{(2 N+3) n}$ and where $S, Q, R$ are in $\mathbb{S}_{+}^{n}$. This new functional is defined using the augmented vector $\tilde{x}_{N}$ defined by

$$
\tilde{x}_{N}^{T}(t)=\left[\begin{array}{lll}
x_{t}^{T}(0) & h(t) \phi_{1, N}^{T}(t) & \left(h_{2}-h(t)\right) \phi_{2, N}^{T}(t)
\end{array}\right] .
$$

where

$$
\begin{aligned}
& \phi_{1, N}(t)=\frac{1}{h(t)} \int_{-h(t)}^{0} \mathbb{L}_{N}\left(\frac{s+h(t)}{h(t)}\right) x_{t}(s) \mathrm{d} s, \\
& \phi_{2, N}(t)=\frac{1}{h_{2}-h(t)} \int_{-h_{2}}^{-h(t)} \mathbb{L}_{N}\left(\frac{s+h_{2}}{h_{2}-h(t)}\right) x_{t}(s) \mathrm{d} s .
\end{aligned}
$$

This functional generalizes the one that has been defined in [18] for time-varying delay systems. Indeed selecting $N=0$ in (8) allows retrieving the same functional as in [18]. The proposed extension to time-varying delays is not an easy task since the time-varying delay appears in the definition of $\phi_{1, N}$ and $\phi_{2, N}$. To achieve this goal, several technical problems arise for the consideration of the time-varying delay.

- The first difficulty refers to the exhibition of LMI conditions to prove the positive definiteness of $V_{N}$. A first solution would be to impose that $P_{N}, S, Q$ and $R$ are positive definite. However, this might be too conservative and we look forward a tighter condition which relies on an extensive use of the Bessel Legendre inequality [19] together with an improved version of the reciprocally convex combination lemma [16].

- In order to satisfy the requirements of the LyapunovKrasovskii theorem, we must also show that the functional $V_{N}$ is upper bounded by some function of the state of the time-delay systems. The consideration of $\phi_{1, N}$ and $\phi_{2, N}$ in the first quadratic term of $V_{N}$ makes this step unclear.

- The computation of the time-derivative of $V_{N}$ requires to express the time-derivative of $\phi_{1, N}$ and $\phi_{2, N}$. This step is also not straightforward and requires a dedicated development.

\section{Preliminary Results}

\section{A. Delay-dependent reciprocally convex inequality}

In the two first steps, we will use an improved version of the reciprocally convex combination lemma originally presented in [16]. The following lemma relaxes the inequality provided in [16] through the introduction of additional matrices variables. 
Lemma 2: Let $n$ be a positive integer and $R_{1}, R_{2}$ be in $\mathbb{S}_{+}^{n}$. If there exist $X_{1}, X_{2}$ in $\mathbb{S}^{n}$ and $Y_{1}, Y_{2}$ in $\mathbb{R}^{n \times n}$ such that

$$
\left[\begin{array}{cc}
R_{1} & 0 \\
0 & R_{2}
\end{array}\right]-\alpha\left[\begin{array}{cc}
X_{1} & Y_{1} \\
Y_{1}^{T} & 0
\end{array}\right]-(1-\alpha)\left[\begin{array}{cc}
0 & Y_{2} \\
Y_{2}^{T} & X_{2}
\end{array}\right] \succeq 0
$$

holds for $\alpha=0,1$, then, the following inequality

$$
\left[\begin{array}{cc}
\frac{1}{\alpha} R_{1} & 0 \\
0 & \frac{1}{1-\alpha} R_{2}
\end{array}\right] \succeq\left[\begin{array}{cc}
R_{1} & 0 \\
0 & R_{2}
\end{array}\right]+(1-\alpha)\left[\begin{array}{cc}
X_{1} & Y_{2} \\
Y_{2}^{T} & 0
\end{array}\right]+\alpha\left[\begin{array}{cc}
0 & Y_{1} \\
Y_{1}^{T} & X_{2}
\end{array}\right]
$$

holds for all $\alpha \in(0,1)$.

Proof: The proof is given in the appendix.

Remark 1: As noticed in [28], the additional variables $X_{1}$ and $X_{2}$ can be removed by taking $X_{1}=R_{1}-Y_{1} R_{2}^{-1} Y_{1}^{T}$ and $X_{2}=R_{2}-Y_{2}^{T} R_{1}^{-1} Y_{2}$, which obviously verifies conditions (10). This manipulation allows a notable reduction of the numerical complexity.

The particular selection in the previous lemma with $X_{1}=$ $X_{2}=0$ and $Y_{1}=Y_{2}=Y$ leads to the original reciprocally convex combination lemma [16]. Lemma 2 refines the original convex combination lemma since it allows obtaining a lower bound which depends explicitly on $\alpha$, which will be related to the time-varying delay $h(t)$.

\section{B. Bessel-Legendre inequality}

The Bessel-Legendre inequality is stated in the next lemma.

Lemma 3: For a given function $x$ in $\mathcal{L}_{2}\left([a, b] \rightarrow \mathbb{R}^{n}\right)$, any scalars $a<b$ and any matrix $R \in \mathbb{S}_{+}^{n}$ the inequality

$$
\int_{a}^{b} x^{T}(s) R x(s) \mathrm{d} s \geq(b-a) \phi_{N}^{T} \tilde{R}_{N} \phi_{N}
$$

holds, for any positive integer $N \geq 0$ where

$$
\begin{aligned}
\phi_{N} & =\frac{1}{b-a} \int_{a}^{b} \mathbb{L}_{N}\left(\frac{s-a}{b-a}\right) x(s) \mathrm{d} s \\
\tilde{R}_{N} & =\operatorname{diag}(R, 3 R, \ldots,(2 N-1) R) .
\end{aligned}
$$

Proof : As in [19], [21], the proof of this lemma is based on the function $z_{n}:\left[\begin{array}{ll}a & b\end{array}\right] \rightarrow \mathbb{R}^{n}$ given by $z_{N}(s)=R x(s)-\mathbb{L}_{N}^{T}\left(\frac{s-a}{b-a}\right) \tilde{R}_{N} \phi_{N}$, where we recall that $\phi_{N}=\frac{1}{b-a} \int_{a}^{b} \mathbb{L}_{N}\left(\frac{s-a}{b-a}\right) x(s) \mathrm{d} s$. This function $z_{N}$ can be viewed as the error between the function $x$ and the best polynomial approximation of $x$ according the inner product. The inequality results from the expansion of $\int_{a}^{b} z_{N}^{T}(s) R^{-1} z_{N}(s) \mathrm{d} s$, which is positive.

Remark 2: The previous inequality includes Jensen's inequality [7], the Wirtinger-based integral inequality and the auxiliary-function based integral inequality [17] as particular cases when $N=0,1,2$, respectively. It is also worth noting that the Parseval identity ensures that the proposed inequality becomes asymptotically non conservative when $N$ goes to infinity.

\section{Stability ANALYSiS}

In order to simplify the exposure of this section, the stability analysis is divided into three parts. The first part deals with the positive definiteness of the functional. The second part aims at obtaining LMI conditions to guarantee the negative definiteness of the derivative of the functional along the trajectories of the system. The last part resumes the main result of the paper.

\section{A. Positive definiteness of $V_{N}$}

Lemma 4: For a given positive integer $N \geq 0$, assume that there exist $P_{N} \in \mathbb{S}^{(2 N+3) n}, S, Q, R \in \mathbb{S}_{+}^{n}$ and a matrix $U \in$ $\mathbb{R}^{n(N+1) \times n(N+1)}$ such that the following LMIs

$$
\Phi_{N}\left(h_{2}\right)=P_{N}+\frac{1}{h_{2}}\left[\begin{array}{cc}
0 & 0 \\
0 & \Phi_{N}^{0}
\end{array}\right] \succ 0, \Phi_{N}^{0}=\left[\begin{array}{cc}
\tilde{S}_{N} & U \\
U^{T} & \tilde{Q}_{N}
\end{array}\right] \succeq 0
$$

are satisfied, where $\tilde{S}_{N}=\operatorname{diag}(S, 3 S, \ldots,(2 N+1) S)$, and $\tilde{Q}_{N}=\operatorname{diag}(Q, 3 Q, \ldots,(2 N+1) Q)$. Then, there exist $\epsilon_{1}>0$ and $\epsilon_{2}>0$ such that the functional $V_{N}\left(x_{t}, \dot{x}_{t}\right)$ satisfies

$$
\epsilon_{1}\left|x_{t}(0)\right|^{2} \leq V_{N}\left(x_{t}, \dot{x}_{t}\right) \leq \epsilon_{2}\left\|x_{t}\right\|_{W}^{2}
$$

where $\left\|x_{t}\right\|_{W}=\max _{s \in\left[-h_{2}, 0\right]}\left|x_{t}(s)\right|+\left(\int_{-h_{2}}^{0}\left|\dot{x}_{t}(s)\right|^{2} \mathrm{~d} s\right)^{\frac{1}{2}}$ (see e.g. [3]).

Proof: The objective of the next developments is to find a lower and an upper bounds of $V_{N}$. In order to derive the first lower bound (i.e. the existence of $\epsilon_{1}$ ), one may apply Lemma 3 to the second and third terms of the functional, which can be done since the matrices $S$ and $Q$ are symmetric positive definite. Hence, applying Lemma 3 to the order $N$ yields

$$
\begin{aligned}
\int_{t-h}^{t} x^{T}(s) S x(s) \mathrm{d} s & \geq h \phi_{1, N}^{T}(t) \tilde{S}_{N} \phi_{1, N}(t) \\
\int_{t-h_{2}}^{t-h} x^{T}(s) Q x(s) \mathrm{d} s & \geq\left(h_{2}-h\right) \phi_{2, N}^{T}(t) \tilde{Q}_{N} \phi_{2, N}(t),
\end{aligned}
$$

which ensures the following inequality

$$
\begin{aligned}
V_{N}\left(x_{t}, \dot{x}_{t}\right) \geq & \tilde{x}_{N}(t)\left(P_{N}+\left[\begin{array}{ccc}
0 & 0 & 0 \\
0 & \frac{1}{h} \tilde{S}_{N} & 0 \\
0 & 0 & \frac{1}{h_{2}-h} \tilde{Q}_{N}
\end{array}\right]\right) \tilde{x}_{N}(t) \\
& +h_{2} \int_{t-h_{2}}^{t} \int_{\theta}^{t} \dot{x}^{T}(s) R \dot{x}(s) \mathrm{d} s \mathrm{~d} \theta .
\end{aligned}
$$

If there exists a matrix $U$ in $\mathbb{R}^{n(N+1) \times n(N+1)}$ such that $\left[\begin{array}{cc}\tilde{S}_{N} & U \\ U^{T} & \tilde{Q}_{N}\end{array}\right] \succeq 0$, then Lemma 2, with $X_{1}=X_{2}=0$ and $Y_{1}=Y_{2}=U$ ensures, together with $R \succ 0$ that $V\left(x_{t}, \dot{x}_{t}\right) \geq \tilde{x}_{N}^{T}(t) \Phi_{N}\left(h_{2}\right) \tilde{x}_{N}(t)$. Therefore, if $\Phi_{N}\left(h_{2}\right) \succ 0$ and $R \succ 0$, then there exists $\epsilon_{1}>0$ such that $V_{N}\left(x_{t}, \dot{x}_{t}\right) \geq$ $\epsilon_{1}\left|x_{t}(0)\right|$, which concludes on the first inequality. To prove the second inequality, there exists a sufficiently large scalar $\eta>0$ such that $P_{N} \prec \eta \operatorname{diag}\left(I, \mathcal{I}_{N}, \mathcal{I}_{N}\right)$ where $\mathcal{I}_{N}=$ $\operatorname{diag}(I, 3 I, \ldots,(2 N+1) I)$. It thus holds

$$
\begin{aligned}
\xi_{N}^{T}(t) P_{N} \xi_{N}(t) \leq & \eta\left|x_{t}(0)\right|^{2}+\eta h^{2}(t) \phi_{1, N}^{T}(t) \mathcal{I}_{N} \phi_{1, N}(t) \\
& +\eta\left(h_{2}-h(t)\right)^{2} \phi_{2, N}^{T}(t) \mathcal{I}_{N} \phi_{2, N}(t) .
\end{aligned}
$$

Using Lemma 3, we obtain

$$
\begin{aligned}
\xi_{N}^{T}(t) P_{N} \xi_{N}(t) \leq & \eta\left|x_{t}(0)\right|^{2}+\eta h(t) \int_{t-h(t)}^{t}|x(s)|^{2} \mathrm{~d} s \\
& +\eta\left(h_{2}-h(t)\right) \int_{t-h(t)}^{t-h_{2}}|x(s)|^{2} \mathrm{~d} s \\
\leq & \eta\left|x_{t}(0)\right|^{2}+\eta h_{2} \int_{t-h_{2}}^{t}|x(s)|^{2} \mathrm{~d} s .
\end{aligned}
$$


Re-injecting this inequality into the definition of the functional $V_{N}$, we can easily obtain that there exists $\epsilon_{2}>0$, such that $V_{N}\left(x_{t}, \dot{x}_{t}\right) \leq \epsilon_{2}\left\|x_{t}\right\|_{W}^{2}$.

\section{B. Negative definiteness of the derivative of $V_{N}$}

The main result of this section is given below

Lemma 5: For a given positive integer $N \geq 0$, assume that there exist $P_{N} \in \mathbb{S}^{(2 N+3) n}, S, Q, R \in \mathbb{S}_{+}^{n}$ and $Y_{1}, Y_{2} \in$ $\mathbb{R}^{(N+2) n \times(N+2) n}$, such that ${ }^{1}$

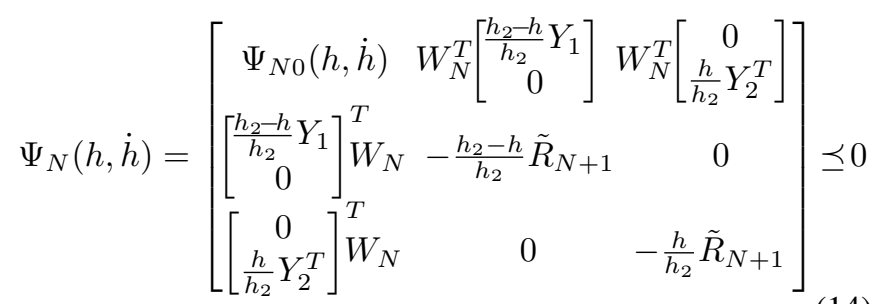

at the vertices of $\mathcal{H}$, where

$$
\begin{aligned}
& \Psi_{N 0}(h, \dot{h})=\operatorname{He}\left(G_{N}^{T}(h) P_{N}\left(J_{N}+\dot{h} H_{N}\right)\right) \\
& +\Sigma_{N}(\dot{h})+h_{2}^{2} F_{N}^{T} R F_{N}-W_{N}^{T} \Xi_{N}(h) W_{N}, \\
& \tilde{R}_{N+1} \quad=\operatorname{diag}(R, 3 R, \ldots,(2 N+1) R,(2 N+3) R) \text {, } \\
& F_{N} \quad=\left[\begin{array}{lllll}
A & A_{d} & 0 & 0 & 0
\end{array}\right] \text {, } \\
& G_{N}(h)=\left[\begin{array}{ccccc}
I & 0 & 0 & 0 & 0 \\
0 & 0 & 0 & h I_{n N} & 0 \\
0 & 0 & 0 & 0 & \left(h_{2}-h\right) I_{n N}
\end{array}\right] \text {, } \\
& H_{N}=\left[\begin{array}{cccccc}
0 & 0 & 0 & 0 & 0 \\
0 & \overline{\mathbf{1}}_{N} & 0 & \bar{\Gamma}_{N}-\Theta_{N} & 0 \\
0 & -\mathbf{1}_{N} & 0 & 0 & \Theta_{N}
\end{array}\right] \text {, } \\
& J_{N} \quad=\left[\begin{array}{ccccc}
A & A_{d} & 0 & 0 & 0 \\
\mathbf{1}_{N} & -\overline{\mathbf{1}}_{N} & 0 & -\bar{\Gamma}_{N} & 0 \\
0 & \mathbf{1}_{N} & -\overline{\mathbf{1}}_{N} & 0 & -\bar{\Gamma}_{N}
\end{array}\right], \\
& \Sigma_{N}(\dot{h})=\operatorname{diag}(S,-(1-\dot{h})(S-Q),-Q, 0,0), \\
& W_{N} \quad=\left[\begin{array}{ccccc}
\mathbf{1}_{N+1} & -\overline{\mathbf{1}}_{N+1} & 0 & -\Gamma_{N+1} & 0 \\
0 & \mathbf{1}_{N+1} & -\overline{\mathbf{1}}_{N+1} & 0 & -\Gamma_{N+1}
\end{array}\right], \\
& \Xi_{N}(h)=\left[\begin{array}{cc}
\tilde{R}_{N+1} & 0 \\
0 & \tilde{R}_{N+1}
\end{array}\right]+\frac{h_{2}-h}{h_{2}}\left[\begin{array}{cc}
\tilde{R}_{N+1} & Y_{2} \\
Y_{2}^{T} & 0
\end{array}\right]+\frac{h}{h_{2}}\left[\begin{array}{cc}
0 & Y_{1} \\
Y_{1}^{T} & \tilde{R}_{N+1}
\end{array}\right] \text {, }
\end{aligned}
$$

with $\mathbf{1}_{N+1}, \overline{\mathbf{1}}_{N+1}, \Gamma_{N}, \bar{\Gamma}_{N}$ and $\Theta_{N}$ are given in (5), (6) and (6), respectively. Then, there exists $\epsilon_{3}>0$ such that the functional $V_{N}\left(x_{t}, \dot{x}_{t}\right)$ satisfies $\dot{V}_{N}\left(x_{t}, \dot{x}_{t}\right) \leq-\epsilon_{3}\left\|x_{t}(0)\right\|^{2}$.

Proof: Before entering into the details of this proof, two preliminary results are proposed for the sake of simplicity. These results use an augmented vector, defined for $N \geq 0$ by

$\xi_{N}^{T}(t)=\left[\begin{array}{lllll}x_{t}^{T}(0) & x_{t}^{T}(-h) & x_{t}^{T}\left(-h_{2}\right) & \phi_{1, N}^{T}(t) & \phi_{2, N}^{T}(t)\end{array}\right]$.

Proposition 6: For a given $N>0$, the time-derivative of the vector $\tilde{x}_{N}$ is expressed using matrices $H_{N}$ and $J_{N}$ given in (15) by $\dot{\tilde{x}}_{N}(t)=\left(J_{N}+\dot{h} H_{N}\right) \xi_{N}(t)$.

Proposition 7: For any function $x$ such that $\dot{x}$ is in $\mathcal{L}_{2}$, any scalar $h_{2}>0$, any integer $N \geq 0$ and any matrix $R \in \mathbb{S}_{+}^{n}$, then the inequality

$h_{2} \int_{-h_{2}}^{0} \dot{x}_{t}^{T}(s) R \dot{x}_{t}(s) \mathrm{d} s \geq \xi_{N}^{T}(t) W_{N}^{T}\left[\Xi_{N}(h)-\Xi_{N 0}(h)\right] W_{N} \xi_{N}(t)$

${ }^{1}$ Conditions (14) are formally strict inequalities for all delays $h$ different from 0 and $h_{2}$. Due to the cases $h=0$ and $h=h_{2}$, some rows and columns might be zero, which implies the non strict inequality (14). holds for any delay $h \in\left[0, h_{2}\right]$ and for any matrices $Y_{1}, Y_{2} \in$ $\mathbb{R}^{(N+2) n \times(N+2) n}$, where $\Xi_{N}(h)$ and $W_{N}$ are defined in (15) and where $\Xi_{N 0}(h)=\left[\begin{array}{cc}\frac{h_{2}-h}{h_{2}} Y_{1} \tilde{R}_{N+1}^{-1} Y_{1}^{T} & 0 \\ 0 & \frac{h}{h_{2}} Y_{2}^{T} \tilde{R}_{N+1}^{-1} Y_{2}\end{array}\right]$. The proof of these two propositions are provided in the appendix. The computation of the derivative of $V_{N}$ leads to

$$
\begin{aligned}
\dot{V}_{N}\left(x_{t}, \dot{x}_{t}\right)= & \operatorname{He}\left(\tilde{x}_{N}^{T}(t) P_{N} \dot{\tilde{x}}_{N}(t)\right)+x_{t}^{T}(0) S x_{t}(0) \\
& -(1-\dot{h}) x_{t}^{T}(-h)(S-Q) x_{t}(-h) \\
& -x_{t}^{T}\left(-h_{2}\right) Q x_{t}\left(-h_{2}\right)+h_{2}^{2} \dot{x}_{t}^{T}(0) R \dot{x}_{t}(0) \\
& -h_{2} \int_{-h_{2}}^{0} \dot{x}_{t}^{T}(s) R \dot{x}_{t}(s) \mathrm{d} s .
\end{aligned}
$$

Our objective is to derive an upper bound of $\dot{V}_{N}$ expressed as a quadratic form using the augmented vector $\xi_{N}(t)$. Using the matrices $G_{N}(h), F_{N}$ and $\Sigma_{N}(\dot{h})$ defined in (15), the derivative of $V_{N}$ along the trajectories of the system can be rewritten as

$$
\begin{aligned}
\dot{V}_{N}\left(x_{t}, \dot{x}_{t}\right)= & \operatorname{He}\left(\xi_{N}^{T}(t) G_{N}^{T}(h) P_{N} \dot{\tilde{x}}_{N}(t)\right) \\
& +\xi_{N}^{T}(t)\left[h_{2}^{2} F_{N}^{T} R F_{N}+\Sigma_{N}(h)\right] \xi_{N}(t) \\
& -h_{2} \int_{-h_{2}}^{0} \dot{x}_{t}^{T}(s) R \dot{x}_{t}(s) \mathrm{d} s .
\end{aligned}
$$

Using Propositions 6 and 7, the derivative of the $V_{N}$ along the trajectories of the system satisfies

$$
\begin{gathered}
\dot{V}_{N}\left(x_{t}, \dot{x}_{t}\right) \leq \xi_{N}^{T}(t)\left[\operatorname{He}\left(G_{N}^{T}(h) P_{N}\left(J_{N}+\dot{h} H_{N}\right)\right)+\Sigma_{N}(\dot{h})\right. \\
\left.+h_{2}^{2} F_{N}^{T} R F_{N}-W_{N}^{T}\left[\Xi_{N}(h)-\Xi_{N 0}(h)\right] W_{N}\right] \xi_{N}(t) \\
=\xi_{N}^{T}(t)\left(\Psi_{N 0}(h, \dot{h})+W_{N}^{T} \Xi_{N 0}(h) W_{N}\right) \xi_{N}(t) .
\end{gathered}
$$

It is then easy to see that the matrix $\Psi_{N 0}(h, \dot{h})+$ $W_{N}^{T} \Xi_{N 0}(h) W_{N}$ is multi-affine with respect to $h$ and $\dot{h}$ and consequently convex in these two parameters. Hence, if the LMIs (14) are satisfied at the vertices of $\mathcal{H}$, there are also verified for any $(h, \dot{h}) \in \mathcal{H}$ and there exists a sufficiently small $\epsilon_{3}$ such that $\Phi_{N}(h, \dot{h})-\epsilon_{3}\left[\begin{array}{ll}I & 0 \\ 0 & 0\end{array}\right] \prec 0$, which allows concluding the proof.

\section{Main result}

Lemma 4 and 5 allow us to state the main result of this paper, given below.

Theorem 8: For a given positive integer $N \geq 0$, assume that there exist a matrix $P_{N} \in \mathbb{S}^{(2 N+3) n}$, matrices $S, Q, R \in \mathbb{S}_{+}^{n}$ and two matrices $Y_{1}, Y_{2} \in \mathbb{R}^{n(N+2) \times n(N+2)}$ and $U \in \mathbb{R}^{n(N+1) \times n(N+1)}$ such that LMI $\Phi_{N}\left(h_{2}\right) \succ 0$, $\Phi_{N}^{0} \succ 0$ and $\Psi_{N}(h, \dot{h}) \prec 0$ are satisfied for all $(h, \dot{h}) \in \mathcal{H}$, then system (1) is asymptotically stable for any time-varying delay $h$, satisfying (2).

Proof : According to Lemmas 4 and 5, provided that the LMI conditions hold, the functional $V_{N}$ satisfies the following inequalities $\epsilon_{1}\left|x_{t}(0)\right|^{2} \leq V_{N}\left(x_{t}, \dot{x}_{t}\right) \leq \epsilon_{2}\left\|x_{t}\right\|_{W}^{2}$ and $\dot{V}_{N}\left(x_{t}, \dot{x}_{t}\right) \leq-\epsilon_{3}\left\|x_{t}(0)\right\|^{2}$. These inequalities ensures the asymptotic stability of the delay system (1) for any time-varying time delay $h$, which satisfies $(h, \dot{h}) \in \mathcal{H}$, by application of the extended Lyapunov-Krasovskii theorem (see e.g. [3]) 


\section{Hierarchy of LMI conditions}

Following the results presented in [19], [21] dealing with systems subject to constant discrete and distributed delays and also taking advantages of the Bessel-Legendre framework, the LMI conditions presented in Theorem 8 form a hierarchy. In other words, it means that increasing $N$ can only reduce the conservatism. This is summarized in the following theorem.

Theorem 9: For a given time-delay system (1) and a given allowable delay set $\mathcal{H}$, if there exists a positive integer $N_{0}$ such that the LMI conditions of Theorem 8 are satisfied at the order $N_{0}$, then the same LMIs are also verified for all integer $N \geq N_{0}$.

Proof: Assume that the LMI condition of Theorem 8 are verified at a given order $N_{0} \in \mathbb{N}$. Then there exist matrices $P_{N_{0}}, S, Q, R, U$ and $Y_{1}, Y_{2}$ of appropriate dimensions such that the following inequalities are verified for all $(h, \dot{h}) \in \mathcal{H}$

$$
\Phi_{N_{0}}\left(h_{2}\right) \succ 0, \quad \Phi_{N_{0}}^{0} \succ 0, \quad \Psi_{N_{0}}(h, \dot{h}) \prec 0 .
$$

To prove that the same LMIs also hold at the order $N_{0}+1$, it suffices to keep the same matrices $S, Q, R$ and to introduce $P_{N_{0}+1}=\left[\begin{array}{rr}P_{N_{0}} & 0 \\ 0 & 0\end{array}\right], U^{+}=\left[\begin{array}{ll}U & 0 \\ 0 & 0\end{array}\right]$ and $Y_{i}^{+}=\left[\begin{array}{cc}Y_{i} & 0 \\ 0 & 0\end{array}\right]$, for $i=1,2$. Using these definitions and using some matrix congruence, we obtain the following equivalences

$$
\begin{aligned}
\Phi_{N_{0}+1}\left(h_{2}\right) \succ 0 & \Leftrightarrow\left[\begin{array}{ccc}
\Phi_{N_{0}}\left(h_{2}\right) & 0 & 0 \\
0 & \left(2 N_{0}+3\right) S & 0 \\
0 & 0 & \left(2 N_{0}+3\right) Q
\end{array}\right] \succ 0, \\
\Phi_{N_{0}+1}^{0} \succ 0 & \Leftrightarrow\left[\begin{array}{ccc}
\Phi_{N_{0}}^{0} & 0 & 0 \\
0 & \left(2 N_{0}+3\right) S & 0 \\
0 & 0 & \left(2 N_{0}+3\right) Q
\end{array}\right] \succ 0, \\
\Psi_{N_{0}+1}(h, \dot{h}) \prec 0 & \Leftrightarrow\left[\begin{array}{ccc}
\Psi_{N_{0}}(h, \dot{h}) & 0 & 0 \\
0 & -\bar{R}_{N_{0}} & 0 \\
0 & 0 & -\bar{R}_{N_{0}}
\end{array}\right] \preceq 0,
\end{aligned}
$$

where $\quad \bar{R}_{N_{0}} \quad=\quad \operatorname{diag}(R,(2 N+$ 5) $\left.\left(\Gamma_{N_{0}+2}\right)_{N_{0}+2}^{T} R\left(\Gamma_{N_{0}+2}\right)_{N_{0}+2}\right)$, with $\left(\Gamma_{N_{0}+2}\right)_{N_{0}+2}$ being the $n$ last lines of the matrix $\Gamma_{N_{0}+2}$. Therefore, there exists a solution to the LMI problem at the order $N_{0}+1$. By induction, we can easily show that for any integer $N \geq N_{0}$, there also exists a solution to the same LMI problem at the order $N$, which concludes the proof.

This theorem can be interpreted as an inclusion of the inner allowable delay sets obtained through Theorem 8 .

\section{Allowable Delay Sets}

In Theorem 8, the resulting LMIs are multi-affine in the delay parameters $h$ and $\dot{h}$. Then, in order to get tractable numerical conditions, it suffices to consider that $\mathcal{H}$ is a polyhedral set or if it is not the case to embed it by a polytopic outer approximation. In the literature, only a few attention has been taken on the definition of the set $\mathcal{H}$. In the following we will recall, in a first step, the main characterization that has been considered in the literature. Then, based on an discussion regarding this first set, an alternative allowable delay set is introduced in order to provided a more natural and accurate characterization of the delay function. A consequence of this selection is a notable modification of the numerical results.

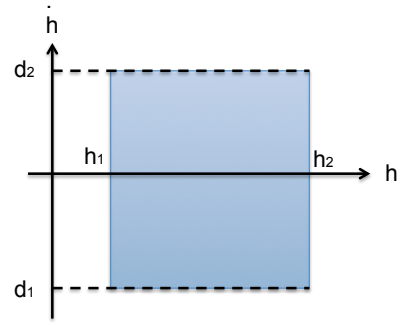

(a) Graph representing $\mathcal{H}_{1}$

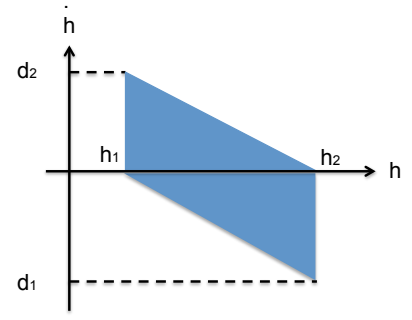

(b) Graph representing $\mathcal{H}_{2}$
Fig. 1. Graphical illustration of the delay set $\mathcal{H}_{1}$ (a) and $\mathcal{H}_{2}$ (b).

\section{A. Usual assumption on the delay set $\mathcal{H}$}

The usual formulation of the delay set is as follows. Consider some scalars $0 \leq h_{2}$ and $d_{1} \leq 0 \leq d_{2}$ and assumed that the delay function satisfies:

$$
(h, \dot{h}) \in \mathcal{H}_{1}=\left[0, h_{2}\right] \times\left[d_{1}, d_{2}\right] .
$$

This set is the usual characterization of the delay function. Indeed, it allows the delay function to take any values in the interval $\left[0, h_{2}\right]$ while its derivative belongs to the interval $\left[d_{1}, d_{2}\right]$. Notice that it is implicitly assumed that $h$ and $\dot{h}$ are independent parameters. However this definition requires that the LMI are satisfied whenever $h=h_{2}$ and $\dot{h}>0$ or $h=h_{1}$ and $\dot{h}<0$. These situations contradict the fact that $h_{1}$ and $h_{2}$ are respectively the upper and the lower bounds of the delay.

\section{B. Refined characterization of the allowable delay set}

As pointed out in the previous section, the polytopic modeling of the allowable delay set allows to exclude some subsets of $\mathcal{H}_{1}$. A first direction is to note that when $h=h_{2}$ (or $h=0$ ), its derivative cannot be positive (negative). Following this comment, we introduce the new allowable delay set $\mathcal{H}_{2}$ described as follows. Consider some scalars $0 \leq h_{2}$ and $d_{1}<0<d_{2}$ and we assume that the delay function satisfies:

$$
(h, \dot{h}) \in \mathcal{H}_{2}=\mathcal{C} o\left\{(0,0),\left(h_{1}, d_{2}\right),\left(h_{2}, 0\right),\left(h_{2}, d_{1}\right)\right\} .
$$

A graphical interpretation of the sets $\mathcal{H}_{1}$ and $\mathcal{H}_{2}$ are depicted in Figure 1, where it is shown that, for fixed values of $h_{2}, d_{1}, d_{2}, \mathcal{H}_{2}$ is included in $\mathcal{H}_{1}$. In the next section which presents the application of Theorem 8 on an example, the use of an accurate selection of the allowable delay set notably modifies the numerical results, as expected and including additional information on the delay functions may enlarge these results.

\section{NUMERICAL EXAMPLE}

In this section, we will consider the linear time-delay system (1) with the matrices

$$
\dot{x}(t)=\left[\begin{array}{cc}
-2 & 0 \\
0 & -0.9
\end{array}\right] x(t)+\left[\begin{array}{cc}
-1 & 0 \\
-1 & -1
\end{array}\right] x(t-h(t)) .
$$

This system is a well-known delay dependent stable system, that is the delay free system is stable and the maximum allowable delay $h_{\max }=6.1725$ can be easily computed by delay sweeping techniques (see for instance [7]). Of course, in the time-varying delay case, such sweeping techniques cannot be considered. 


\begin{tabular}{|c||c|c|c|c|c|c|c|c|}
\hline$d_{2}$ & 0 & 0.1 & 0.2 & 0.5 & 0.8 & 1 & Number of decision variables & Number of LMIs \\
\hline \hline$[4],[24]$ & 4.47 & 3.60 & 3.03 & 2.00 & 1.36 & 0.99 & $7 n^{2}+3 n / 2.5 n^{2}+3.5 n$ & $2 / 2$ \\
\hline$[8]$ & 4.47 & 3.60 & 3.03 & 2.04 & 1.49 & 1.34 & $9 n^{2}+3 n$ & $1 / 1$ \\
\hline$[23]$ & 4.47 & 3.61 & 3.04 & 2.07 & 1.59 & 1.52 & $7 n^{2}+n$ & 2 \\
\hline$[16]$ & 4.47 & 3.65 & 3.16 & 2.33 & 1.93 & 1.86 & $3 n^{2}+2 n$ & 2 \\
\hline$[11]$ & 6.11 & 4.71 & 3.80 & 2.28 & 1.60 & 1.36 & $1.5 n^{2}+9 n+9$ & 1 \\
\hline$[1]$ & 6.11 & 4.79 & 3.99 & 2.68 & 1.95 & 1.60 & $22 n^{2}+8 n$ & 4 \\
\hline$[26]\left(N^{*}=3\right)$ & 5.90 & 4.62 & 3.76 & 2.44 & 2.07 & 2.07 & $17 n^{2}+5 n$ & 2 \\
\hline$[18]$ & 6.05 & 4.70 & 3.83 & 2.42 & 2.08 & 2.04 & $10 n^{2}+3 n$ & 5 \\
\hline$[12]$ & 6.05 & 4.81 & 4.10 & 3.06 & 2.61 & - & $27 n^{2}+17 n$ & 5 \\
\hline$[25]$ & 6.05 & 4.78 & 4.06 & 3.05 & 2.61 & - & $65 n^{2}+11 n$ & 4 \\
\hline$[27]($ Th2.C2) & 6.16 & 4.71 & 3.85 & 2.60 & 2.37 & 2.31 & $23 n^{2}+4 n$ & 5 \\
\hline \hline Th.8 $N=0$ & 6.05 & 4.72 & 3.86 & 2.45 & 2.14 & 2.12 & $14.5 n^{2}+3.5 n$ & 5 \\
\hline Th.8 $N=1$ & 6.16 & 4.80 & 3.99 & 2.79 & 2.42 & 2.33 & $34 n^{2}+5 n$ & 5 \\
\hline Th. $8 N=2$ & 6.1725 & 4.93 & 4.22 & 3.09 & 2.66 & 2.50 & $62.5 n^{2}+6.5 n$ & 5 \\
\hline Th. $N=3$ & 6.1725 & 5.00 & 4.29 & 3.18 & 2.69 & 2.52 & $100 n^{2}+8 n$ & 5 \\
\hline Th. $N=4$ & 6.1725 & 5.01 & 4.29 & 3.19 & 2.70 & 2.52 & $146.5 n^{2}+9.5 n$ & 5 \\
\hline
\end{tabular}

The MAXimal allowable delays $h_{2}$ FOR EXAmple (20) With VARious VAlues of $d_{2}$ AND $d_{1}=-d_{2}$ AND $(h, \dot{h}) \in \mathcal{H}_{1}$.

\section{A. Numerical complexity}

The two last columns of Table I summarize the numerical complexity of various stability conditions from the literature and the ones presented in Theorem 8 by indicating the number of decision variables and the number of LMI conditions to be implemented. For sake of simplicity, it does not include the size of the LMIs.

At this stage, one can note that the complexity of Theorem 8 is similar to the one of existing conditions from the literature, for small values of $N(=0,1)$. For larger values of $N$, the number of decision variables increases in a polynomial way.

\section{B. Numerical results for $\mathcal{H}_{1}$}

Table I shows the numerical results obtained through several criteria using both Lyapunov-Krasovskii and robust analysis for the example. The first rows of Table I present criteria based on Jensen's inequality. The last rows present the numerical results which can be seen as the most recent works on improved integral inequalities including the Wirtinger-based double integral inequality [12], the free-weighting matrix inequality [25]. These contributions are compared with results issued from Theorem 8 .

The numerical results presented in Table I show that the conditions presented in Theorem 8 are less conservative than existing results. It is also worth noting the different levels of conservatism between Theorem 8 are mainly due to the conservatism induced by the reciprocally convex combination lemma of [16] or presented in Lemma 2. This observed conservatism is then partially reduced by the proposed methodology at the price of an increasing numerical burden.

\section{Numerical results for $\mathcal{H}_{2}$}

This subsection presents the numerical results for an alternative allowable delay set, excluding the conflicting point $\left(h_{2}, d_{2}\right)$ and $\left(h_{2}, d_{1}\right)$ which prevents for $h_{2}$ and $h_{1}$ to be the maximum and the minimum of the delay function $h(t)$. Following the discussion presented in Subsection V, we consider here that the delay function $h$ belongs in $\mathcal{H}_{2}$, given in (19). The numerical results presented in Table II show a notable increase of the delay upper bound $h_{2}$ compared to Table I, obtained for $\mathcal{H}_{1}$. One can also see that there is no brutal decrease of the upper bound $h_{2}$ when the bound on the derivative $d_{2}$ is small (see for instance, when the derivative of the delay function become large, the jump from $h_{2}=2.53$ with $\mathcal{H}_{1}$ to $h_{2}=3.70$ with $\mathcal{H}_{2}$ ).

Table II finally demonstrates that the gap between the constant and the time-varying delay case is not only due to the conservatism induced by the choice of the LyapunovKrasovskii functional. The choice of the delay set $\mathcal{H}$ has also a dramatic effect on the results and the conservatism of the method. Indeed, by an accurate modification of the allowable delay set definition, it is possible to notably enlarge the upper bound of the delay $h_{2}$ upperbound. Clearly, there is a room for improvements if we consider a thinner description of the delay set as it has been already noted in [9], [14] for sampled-data systems. It also suggests that a particular attention should be performed when we consider the robust analysis of the LMI conditions.

In order to motivate the notion of allowable delay set, we will consider in the next section, a particular case of delay function, for which several allowable delay sets can be derived.

\section{Numerical results for sinusoidal delays}

It has been shown previously that the selection of the allowable delay set may have a notable impact on the numerical results. In order to illustrate this choice, let consider the particular delay function given by $h(t)=\frac{h_{2}}{2}\left(1+\cos \left(\frac{2 d_{2}}{h_{2}} t\right)\right)$, for all $t \geq 0$, so that the delay function belongs to the convex ellipsoid set defined by $\left(\frac{2 h(t)-h_{2}}{h_{2}}\right)^{2}+\left(\frac{\dot{h}(t)}{d_{2}}\right)^{2} \leq 1$. A first approach would consider that the delay function lies in a 


\begin{tabular}{|c||c|c|c|c|c|c|}
\hline Th.8: $N / d_{M}$ & 0 & 0.1 & 0.2 & 0.5 & 0.8 & 1 \\
\hline \hline 0 & 6.059 & 6.059 & 6.059 & 4.63 & 3.45 & 3.02 \\
\hline 1 & 6.168 & 6.168 & 6.154 & 4.83 & 3.70 & 3.37 \\
\hline 2 & 6.1725 & 6.1725 & 6.164 & 5.07 & 3.94 & 3.58 \\
\hline 3 & 6.1725 & 6.1725 & 6.171 & 5.11 & 4.01 & 3.67 \\
\hline 4 & 6.1725 & 6.1725 & 6.1725 & 5.12 & 4.02 & 3.70 \\
\hline \multicolumn{5}{|c|}{ TABLE II }
\end{tabular}

THE MAXIMAL ALLOWABLE DELAYS $h_{2}$ FOR SYSTEM DESCRIBED IN EXAMPLE (20) SOLVING THEOREM 8 FOR VARIOUS VALUES OF $N$ AND $d_{2}$ AND $d_{1}=-d_{2}$ WITH $(h, \dot{h}) \in \mathcal{H}_{2}$.

polytope of $\mathcal{H}_{1}$-type given by $\left[0, h_{2}\right] \times\left[-d_{2}, d_{2}\right]$. However, it is possible to define other polytopes, still with four vertices, which allow characterizing this class of delay functions. Figure 2 depicts a possible solution obtained by the geometric properties of ellipsoids which does not increase the complexity of the LMI problem. Using the equation of the tangent to an ellipsoid, another polytope is defined, for a given angle $\theta \in[0, \pi / 2)$, by $\mathcal{H}_{\theta}:=\operatorname{Co}\left\{\left(0,-d_{1}(\theta)\right),\left(0, d_{2}(\theta)\right),\left(h_{2}, d_{1}(\theta)\right),\left(h_{2},-d_{2}(\theta)\right)\right\}$, where

$$
d_{1}(\theta)=d_{2} \frac{1-\cos (\theta)}{\sin (\theta)}, \quad d_{2}(\theta)=d_{2} \frac{1+\cos (\theta)}{\sin (\theta)} .
$$

Figure 3 shows how the upper bound of the delay $h_{2}$ varies with the parameter $\theta$ with defines the polytope for various values of $d_{2}$. It can also be seen that the maximal delay bound $h_{2}$ is never obtained when $\theta=\pi / 2$, corresponding to the $\mathcal{H}_{1}$. This demonstrates the relevance of the selection of the allowable delay set.

It would be also possible to refine the selection of the delay set by including other vertices. For instance, one could select the set $\mathcal{H}_{1} \cap \mathcal{H}_{\theta}$, which would introduce two additional vertices.

\section{CONCLUSIONS}

In this paper, we have provided new stability tests for systems subject to a continuous time-varying delay with bounded derivatives. The novelty of this contribution is due to an extensive use of the Bessel-Legendre inequality. The set of LMI conditions forms a hierarchy, which means that increasing the order of $N$ of the Legendre polynomials in the analysis can only improve the numerical results. Compared to existing resulting from the literature, notable improvements of the numerical results have been obtained. The second contribution of the paper relies on the definition of allowable delay sets. It is shown that a slight modification of the definition of the delay set allows to obtain more accurate results. In particular, the upper bound of the delays for slow varying delays is very closed to the one obtained in the constant case.

\section{APPENDIX}

\section{PROOF OF LEMMA 2}

Proof: Following [16], the proof consists in noting that

$$
\left[\begin{array}{cc}
\frac{1}{\alpha} R_{1} & 0 \\
* & \frac{1}{1-\alpha} R_{2}
\end{array}\right]=\left[\begin{array}{cc}
R_{1} & 0 \\
0 & R_{2}
\end{array}\right]+\left[\begin{array}{cc}
\frac{1-\alpha}{\alpha} R_{1} & 0 \\
0 & \frac{\alpha}{1-\alpha} R_{2}
\end{array}\right]
$$

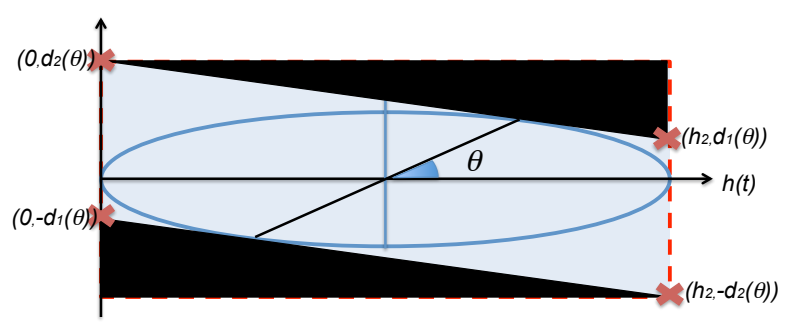

Fig. 2. Graphical illustration of the delay set $\mathcal{H}_{3}$ (a) and $\mathcal{H}_{2}$ (b).

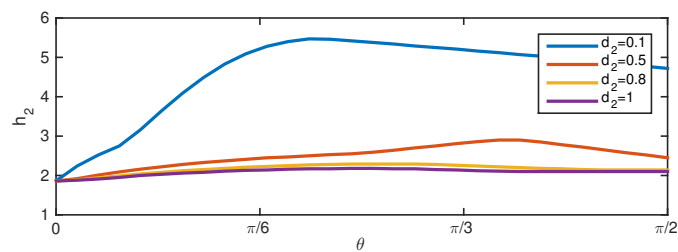

Fig. 3. Graphical illustration of the delay set $\mathcal{H}_{3}$ (a) and $\mathcal{H}_{2}$ (b).

The objective is to find a lower bound of the second term of the right-hand-side of (21). Using a convexity argument, if (10) holds for $\alpha=0,1$, it also holds for any $\alpha$ in $[0,1]$. Then, pre- and post-multiplying (10) by $\left[\begin{array}{cc}\beta I & 0 \\ 0 & \beta^{-1} I\end{array}\right]$, with $\beta=\sqrt{\frac{1-\alpha}{\alpha}}$ yields, for all $\alpha$ in $(0,1)$

$$
\left[\begin{array}{cc}
\frac{1-\alpha}{\alpha} R_{1} & 0 \\
* & \frac{\alpha}{1-\alpha} R_{2}
\end{array}\right] \succeq(1-\alpha)\left[\begin{array}{cc}
X_{1} & Y_{2} \\
Y_{2}^{T} & 0
\end{array}\right]+\alpha\left[\begin{array}{cc}
0 & Y_{1} \\
Y_{1}^{T} & X_{2}
\end{array}\right],
$$

which together with (21) yields the result.

\section{PROOF OF PROPOSITION 6}

Proof : For simplicity, the time argument will be omitted in the next developments. Consider $\phi_{N}=$ $\frac{1}{b-a} \int_{a}^{b} \mathbb{L}_{N}\left(\frac{s-a}{b-a}\right) x(s) \mathrm{d} s$ where $a$ and $b$ are functions of time. In order to simplify the computations, we apply the change of variable $s(u)=(b-a) u+a$ to get $\phi_{N}=\int_{0}^{1} \mathbb{L}_{N}(u) x(s(u)) \mathrm{d} u$ and

$$
\frac{d}{d t}\left[(b-a) \phi_{N}\right]=\dot{a} \psi_{1, N}+(\dot{b}-\dot{a})\left(\psi_{2, N}+\phi_{N}\right)
$$

where $\left.\psi_{1, N}=(b-a) \int_{0}^{1} \mathbb{L}_{N}(u) \dot{x}(s(u))\right) \mathrm{d} u$, and $\psi_{2, N}=(b-$ a) $\int_{0}^{1} u \mathbb{L}_{N}(u) \dot{x}(s(u)) \mathrm{d} u$. An integration by parts yields

$$
\begin{aligned}
& \psi_{1, N}=\mathbb{L}_{N}(1) x(b)-\mathbb{L}_{N}(0) x(a)-\int_{0}^{1} \dot{\mathbb{L}}_{N}(u) x(s(u)) \mathrm{d} u, \\
& \psi_{2, N}=\mathbb{L}_{N}(1) x(b)-\int_{0}^{1} \frac{d}{d u}\left(u \mathbb{L}_{N}(u)\right) x(s(u)) \mathrm{d} u .
\end{aligned}
$$

Rules (6) of the Legendre polynomials yield

$$
\begin{aligned}
\psi_{1, N} & =\mathbf{1}_{\mathbf{N}} x(b)-\overline{\mathbf{1}}_{\mathbf{N}} x(a)-\bar{\Gamma}_{N} \phi_{N}, \\
\psi_{2, N} & =\mathbf{1}_{\mathbf{N}} x(b)-\Theta_{N} \phi_{N}-\phi_{N} .
\end{aligned}
$$

Reinjecting these equations into (22) yields, for all $t \in \mathbb{R}^{+}$,

$$
\begin{aligned}
& \frac{d}{d t}\left[(b-a) \phi_{N}\right]=\dot{a}\left(\mathbf{1}_{\mathbf{N}} x(b)-\overline{\mathbf{1}}_{\mathbf{N}} x(a)-\bar{\Gamma}_{N} \phi_{N}\right) \\
& \quad+(\dot{b}-\dot{a})\left(\mathbf{1}_{\mathbf{N}} x(b)-\Theta_{N} \phi_{N}-\phi_{N}+\phi_{N}\right) \\
& \quad=\dot{b} \mathbf{1}_{\mathbf{N}} x(b)-\dot{a} \overline{\mathbf{1}}_{\mathbf{N}} x(a)-\left(\dot{a} \bar{\Gamma}_{N}+(\dot{b}-\dot{a}) \Theta_{N}\right) \phi_{N} .
\end{aligned}
$$


This previous expression is translated to the vector $\phi_{1, N}$ and $\phi_{2, N}$ by considering $(a, b)=(t-h(t), t)$ and $(a, b)=$ $\left(t-h_{2}, t-h(t)\right)$, respectively. This yields to

$$
\dot{\tilde{x}}_{N}(t)=\left[\begin{array}{lll}
\dot{x}^{T}(t) & \frac{\mathrm{d}}{\mathrm{d} t}\left(h \phi_{1, N}^{T}\right) & \frac{\mathrm{d}}{\mathrm{d} t}\left(\left(h_{2}-h\right) \phi_{2, N}^{T}\right)
\end{array}\right],
$$

with $\dot{x}(t)=A(t)+A_{d} x(t-h)$ and

$$
\begin{aligned}
\frac{\mathrm{d}}{\mathrm{d} t}\left(h \phi_{1, N}\right)= & \mathbf{1}_{\mathbf{N}} x(t)-(1-\dot{h}) \overline{\mathbf{1}}_{\mathbf{N}} x(t-h) \\
& -\left((1-\dot{h}) \bar{\Gamma}_{N}+\dot{h} \Theta_{N}\right) \phi_{1, N} \\
\frac{\mathrm{d}}{\mathrm{d} t}\left(\left(h_{2}-h\right) \phi_{2, N}\right)= & (1-\dot{h}) \mathbf{1}_{\mathbf{N}} x(t-h)-\overline{\mathbf{1}}_{\mathbf{N}} x\left(t-h_{2}\right) \\
& -\left(\bar{\Gamma}_{N}-\dot{h} \Theta_{N}\right) \phi_{2, N}
\end{aligned}
$$

Using matrices $J_{N}$ and $H_{N}$, the previous equations can be summarized as $\dot{\tilde{x}}_{N}=\left(J_{N}+\dot{h} H_{N}\right) \xi_{N}$.

\section{PROOF OF PROPOSITION 7}

Proof: As we mentioned in the introduction, one of the most popular term employed in a Lyapunov-Krasovskii functional leads to an integral quadratic term of the timederivative of $x$. Thus, the first step of the proof consist in particularizing the inequality provided in Lemma 3 to this situation. Consider the integral $h \int_{t-h}^{t} \dot{x}^{T}(s) R \dot{x}(s) \mathrm{d} s$. By application of the Bessel-Legendre inequality in Lemma 3, to the order $N+1$, we obtain

$\int_{t-h_{2}}^{t} \dot{x}^{T}(s) R \dot{x}(s) \mathrm{d} s \geq\left[\begin{array}{c}\tilde{\phi}_{1, N+1} \\ \tilde{\phi}_{2, N+1}\end{array}\right]^{T}\left[\begin{array}{cc}h \tilde{R}_{N+1} & 0 \\ 0 & \left(h_{2}-h\right) \tilde{R}_{N+1}\end{array}\right]\left[\begin{array}{c}\tilde{\phi}_{1, N+1} \\ \tilde{\phi}_{2, N+1}\end{array}\right]$

where

$$
\begin{aligned}
& \tilde{\phi}_{1, N+1}=\frac{1}{h} \int_{-h}^{0} \mathbb{L}_{N+1}\left(\frac{s+h}{h}\right) \dot{x}_{t}(s) \mathrm{d} s \\
& \tilde{\phi}_{2, N+1}=\frac{1}{h_{2}-h} \int_{-h_{2}}^{-h} \mathbb{L}_{N+1}\left(\frac{s+h_{2}}{h_{2}-h}\right) \dot{x}_{t}(s) \mathrm{d} s .
\end{aligned}
$$

An integration by parts and the differentiation rule (6) yield

$$
\begin{aligned}
{\left[\begin{array}{c}
h \tilde{\phi}_{1, N+1} \\
\left(h_{2}-h\right) \tilde{\phi}_{2, N+1}
\end{array}\right] } & =\left[\begin{array}{c}
\mathbf{1}_{\mathbf{N}+\mathbf{1}} x_{t}(0)-\overline{\mathbf{1}}_{\mathbf{N}+\mathbf{1}} x_{t}(-h)-\Gamma_{N+1} \phi_{1, N}, \\
\mathbf{1}_{\mathbf{N}+\mathbf{1}} x_{t}(-h)-\overline{\mathbf{1}}_{\mathbf{N}+\mathbf{1}} x_{t}\left(-h_{2}\right)-\Gamma_{N+1} \phi_{2, N}
\end{array}\right] \\
& =W_{N} \xi_{N}
\end{aligned}
$$

where the matrix $W_{N}$ is given in the statement of the lemma. Combining this expression into (23) yields

$h_{2} \int_{t-h_{2}}^{t} \dot{x}^{T}(s) R \dot{x}(s) \mathrm{d} s \geq \xi_{N}^{T}(t) W_{N}^{T}\left[\begin{array}{ccc}\frac{h_{2}}{h} \tilde{R}_{N+1} & 0 \\ 0 & \frac{h_{2}}{h_{2}-h} \tilde{R}_{N+1}\end{array}\right] W_{N} \xi_{N}(t)$.

We are now in the situation to apply the convex inequality provided in Lemma 2. It implies that, if there exist $X_{1}, X_{2} \in \mathbb{S}^{n N}$ and $Y_{1}, Y_{2} \in \mathbb{R}^{n N \times n N}$ such that the two conditions $\Psi_{N_{0}}^{1} \succ 0$ and $\Psi_{N_{0}}^{2} \succ 0$ hold, then we have $\left[\begin{array}{cc}\frac{h_{2}}{h} \tilde{R}_{N} & 0 \\ 0 & \frac{h_{2}}{h_{2}-h} \tilde{R}_{N}\end{array}\right] \succ \Xi_{N}(h)$. Reinjecting this inequality into the previous equation concludes the proof of the lemma.

\section{REFERENCES}

[1] Y. Ariba, F. Gouaisbaut, and K.H. Johansson. Robust stability of timevarying delay systems: The quadratic separation approach. Asian Journal of Control, 14(5):1205-1214, 2012.

[2] C. Briat. Linear Parameter-Varying and Time-Delay Systems. Analysis, Observation, Filtering and Control, Advanced in Delays and Dynamics, vol. 3. Springer, Hidelberg, 2015.
[3] E. Fridman. Introduction to Time-Delay Systems: Analysis and Control. Birkhauser, Basel, 2014.

[4] E. Fridman and U Shaked. An improved stabilization method for linear time-delay systems. IEEE Trans. on Automatic Control, 47:1931-1937, November 2002.

[5] E. Fridman and U. Shaked. Delay-dependent stability and h control: constant and time-varying delays. Int. J. of Contr., 76(1):48-60, 2003.

[6] E. Fridman and U. Shaked. Input-output approach to stability and $L_{2}$ gain analysis of systems with time-varying delays. Systems and Control Letters, 55(12):1041-1053, 2006.

[7] K. Gu, V.-L. Kharitonov, and J. Chen. Stability of time-delay systems. Birkhauser, 2003.

[8] Y. He, Q. G. Wang, C. Lin, and M. Wu. Delay-range-dependent stability for systems with time-varying delay. Automatica, 43:371-376, 2007.

[9] L. Hetel, C. Fiter, H. Omran, A. Seuret, E. Fridman, J.-P. Richard, and S.I. Niculescu. Recent developments on the stability of systems with aperiodic sampling: an overview. Automatica, 76:309-335, 2017.

[10] X. Jiang and Q.-L. Han. New stability criteria for linear systems with interval time-varying delay. Automatica, 44(10):2680-2685, 2008.

[11] C.Y. Kao and A. Rantzer. Stability analysis of systems with uncertain time-varying delays. Automatica, 43(6):959 - 970, 2007.

[12] O.M. Kwon, M.J. Park, J.H. Park, S.M. Lee, and E.J. Cha. Improved results on stability of linear systems with time-varying delays via Wirtinger-based integral inequality. Journal of the Franklin Institute, 351(12):5386-5398, 2014.

[13] Y.S. Moon, P.G. Park, W.H. Kwon, and Y.S. Lee. Delay-dependent robust stabilization of uncertain state delayed systems. Int. J. of Contr., 74:1447-1455, 2001

[14] P. Naghshtabrizi, J.P. Hespanha, and A.R. Teel. Exponential stability of impulsive systems with application to uncertain sampled-data systems. Systems and Control Letters, 57(5):378-385, 2008.

[15] P.G. Park and J.W. Ko. Stability and robust stability for systems with a time-varying delay. Automatica, 43(10):1855-1858, 2007.

[16] P.G. Park, J.W. Ko, and C. Jeong. Reciprocally convex approach to stability of systems with time-varying delays. Automatica, 47:235-238, 2011.

[17] P.G. Park, W.I. Lee, and S.Y. Lee. Auxiliary function-based integral inequalities for quadratic functions and their applications to time-delay systems. Journal of the Franklin Institute, 352(4):1378-1396, 2015.

[18] A. Seuret and F. Gouaisbaut. Wirtinger-based integral inequality: application to time-delay systems. Automatica, 49(9):2860-2866, 2013.

[19] A. Seuret and F. Gouaisbaut. Hierarchy of LMI conditions for the stability analysis of time delay systems. Systems and Control Letters, 81:1-7, 2015.

[20] A. Seuret and F. Gouaisbaut. Allowable delay sets for the stability analysis of linear time-varying delay systems using a delay-dependent reciprocally convex lemma. 2017.

[21] A. Seuret, F. Gouaisbaut, and Y. Ariba. Complete quadratic Lyapunov functionals for distributed delay systems. Automatica, 62:168-176, 2015.

[22] R. Sipahi, S.-I. Niculescu, C.T. Abdallah, W. Michiels, and K. Gu. Stability and stabilization of systems with time delay. Control Systems, IEEE, 31(1):38-65, 2011.

[23] J. Sun, G.P. Liu, J. Chen, and D. Rees. Improved delay-range-dependent stability criteria for linear systems with time-varying delays. Automatica, 46(2):466 - 470, 2010.

[24] M. Wu, Y. He, J. H. She, and G. P. Liu. Delay-dependent criteria for robust stability of time-varying delay systems. Automatica, 40:14351439, 2004.

[25] H.-B. Zeng, Y. He, M. Wu, and J. She. Free-matrix-based integral inequality for stability analysis of systems with time-varying delay. Automatic Control, IEEE Transactions on, 60(10):2768-2772, 2015.

[26] H.-B. Zeng, Y. He, M. Wu, and S.-P. Xiao. Less conservative results on stability for linear systems with a time-varying delay. Optimal Control Applications and Methods, 34(6):670-679, 2013.

[27] C.-K. Zhang, Y. He, L. Jiang, M. Wu, and H.-B. Zeng. Stability analysis of systems with time-varying delay via relaxed integral inequalities. Systems \& Control Letters, 92:52-61, 2016.

[28] X-M Zhang, G.L. Han, A. Seuret, and F. Gouaisbaut. An improved reciprocally convex inequality and an augmented lyapunov-krasovskii functional for stability of linear systems with time-varying delay. provisionally accepted in Automatica, 2017. 